N. Kenmochi and Y. Mizuta

Nagoya Math. J.

Vol. 59 (1975), 199-215

\title{
POTENTIAL THEORETIC PROPERTIES OF THE GRADIENT OF A CONVEX FUNCTION ON A FUNCTIONAL SPACE
}

\author{
NOBUYUKI KENMOCHI AND YOSHIHIRO MIZUTA
}

\section{Introduction}

In the previous paper [11], introducing the notions of potentials and of capacity associated with a convex function $\Phi$ given on a regular functional space $\mathfrak{X}(X ; \xi)$, we discussed potential theoretic properties of the gradient $\nabla \Phi$ which were originally introduced and studied by Calvert [5] for a class of nonlinear monotone operators in Sobolev spaces. For example:

( i ) The modulus contraction operates.

( ii ) The principle of lower envelope holds.

(iii) The domination principle holds.

(iv) The contraction $T_{k}$ onto the real interval $[0, k](k>0)$ operates.

( $\mathrm{v}$ ) The strong principle of lower envelope holds.

( vi ) The complete maximum principle holds.

In this paper we shall investigate relations among the properties mentioned above. For this purpose, we shall consider an operator $A$ from a subset of $L^{2}(X ; \xi) \cap \mathfrak{X}(X ; \xi)$ into $L^{2}(X ; \xi)$ associated with $\nabla \Phi$ and its resolvent $R_{\lambda}=(I+\lambda A)^{-1}, \lambda>0$.

One aim is to show (in Theorem A) that each of properties (i) (iii) is equivalent to :

(vii) for any $\lambda>0, R_{\lambda}$ is order-preserving in $L^{2}(X ; \xi)$. Another aim is to show (in Theorem B) that if (i) is satisfied, then each of properties (iv) $\sim$ (vi) holds if and only if

(viii) $R_{\lambda}\left(f+T_{k} g\right) \leq R_{\lambda} f+k$ holds for any $\lambda>0$ and any $f, g \in L^{2}(X ; \xi)$.

These assertions are nonlinear analogues of results in the Dirichlet space (cf. Deny [7; Théorèmes 1 et 2] and Itô [8; Theorems 3 and 5]). The crucial step in the proofs is to deduce both (i) and (iv) from (vii)

Received May 7, 1974. 
and (viii). The key tool is the fact that if $f \in L^{2}(X ; \xi)$ and if

$$
\left\{\int_{X} \lambda^{-1}(f-R, f) f d \xi ; \lambda>0\right\}
$$

is bounded, then $f \in \mathfrak{X}(X ; \xi)$. This fact is a nonlinear extension of a result in [7]. At the end of this paper we shall consider the nonlinear contraction semigroup $S=\{S(t) ; t>0\}$ on $L^{2}(X ; \xi)$ generated by $-A$ and show (in Theorem $C$ ) that each of properties (i) $\sim$ (iii) and (vii) is necessary and sufficient for $S$ to be order-preserving in $L^{2}(X ; \xi)$.

\section{§1. Preliminaries}

Let $X$ be a locally compact Hausdorff space with a countable base and $\xi$ be a positive (Radon) measure on $X$. Let $\mathfrak{X}=\mathfrak{X}(X ; \xi)$ be a real reflexive Banach space whose elements are real-valued locally $\xi$-summable functions defined $\xi$-a.e. on $X$. We denote by $\mathfrak{X}^{*}$ the dual space of $\mathfrak{X}$, by $\|u\|$ (resp. $\left\|u^{*}\right\|$ ) the norm of $u \in \mathfrak{X}$ (resp. $u^{*} \in \mathfrak{X}^{*}$ ) and by $\left\langle u^{*}, u\right\rangle$ the value of $u^{*} \in \mathfrak{X}^{*}$ at $u \in \mathfrak{X}$. By “ $\stackrel{s}{\longrightarrow}$ " (resp. “ $\stackrel{w}{\longrightarrow}$ ") we mean the strong (resp. weak) convergence. For functions $u, v \in L_{1 \mathrm{loc}}^{1}(X ; \xi)$, we write $u \vee v$, $u \wedge v, u^{+}$and $u^{-}$for $\max (u, v), \min (u, v), \max (u, 0)$ and $-\min (u, 0)$, respectively, and for a $\xi$-measurable set $S$ in $X$ we write simply " $u \geq v$ (resp. $u=v$ ) on $S$ " for " $u \geq v$ (resp. $u=v$ ) $\xi$-a.e. on $S$ ". Especially we write " $u \geq v$ (resp. $u=v$ )" for " $u \geq v$ (resp. $u=v$ ) on $X$ ".

Throughout this paper, let $1<p<\infty$ and $\Phi$ be a strictly convex function on $\mathfrak{X}$ such that

$$
\left\{\begin{array}{l}
\Phi(0)=0, \\
\Phi(u) \geq C\|u\|^{p}, \quad \text { for any } u \in \mathfrak{X},
\end{array}\right.
$$

where $C$ is a positive constant. Suppose that $\Phi$ is bounded on each bounded subset of $\mathfrak{X}$ and is everywhere differentiable in the sense of Gâteaux, that is, there is an operator $G: \mathfrak{X} \rightarrow \mathfrak{X}^{*}$ such that for any $u$, $v \in \mathfrak{X}$,

$$
\langle G u, v\rangle=\lim _{t \downarrow 0} \frac{\Phi(u+t v)-\Phi(u)}{t} .
$$

This operator $G$ is called the gradient of $\Phi$ and denoted by $\nabla \Phi$. We shall use the following properties of $\Phi$ and $\nabla \Phi$ without proof:

$\left(\Phi_{1}\right) \quad \Phi$ is weakly sequentially lower semicontinuous in $\mathfrak{X}$.

$\left(\Phi_{2}\right) \quad$ Let $u \in \mathfrak{X}$ and $u^{*} \in \mathfrak{X}^{*}$. Then $u^{*}=\nabla \Phi(u)$ if and only if 


$$
\left\langle u^{*}, v-u\right\rangle \leq \Phi(v)-\Phi(u) \quad \text { for any } v \in \mathfrak{X} .
$$

$\left(\Phi_{3}\right) \quad \nabla \Phi$ is strictly monotone, i.e., $\langle\nabla \Phi(u)-\nabla \Phi(v), u-v\rangle>0 \quad$ for any $u, v \in \mathfrak{X}, u \neq v$.

$\left(\Phi_{4}\right) \quad \nabla \Phi$ is bounded, i.e., it maps bounded sets in $\mathfrak{X}$ to bounded sets in $\mathfrak{X}^{*}$.

$\left(\Phi_{5}\right) \quad \nabla \Phi$ is demicontinuous, i.e., if $u_{n} \stackrel{s}{\longrightarrow} u$ in $\mathfrak{X}$ as $n \rightarrow \infty$, then $\nabla \Phi\left(u_{n}\right) \stackrel{w}{\longrightarrow} \nabla \Phi(u)$ in $\mathfrak{X}^{*}$ as $n \rightarrow \infty$.

$\left(\Phi_{6}\right)$ For each $u \in \mathfrak{X},\langle\nabla \Phi(v), v-u\rangle /\|v\| \rightarrow \infty$ as $\|v\| \rightarrow \infty$.

$\left(\Phi_{7}\right)$ For any $u, v \in \mathfrak{X}$,

$$
\Phi(u)-\Phi(v)=\int_{0}^{1}\langle\nabla \Phi(v+t(u-v)), u-v\rangle d t
$$

Remark. $\nabla \Phi$ is one to one and onto. For a proof, see Browder [2; Theorem 3].

LEMMA 1.1. Let $\left\{u_{n}\right\}$ be a sequence in $\mathfrak{X}$ which converges weakly to $u$ in $\mathfrak{X}$. If $\limsup _{n \rightarrow \infty}\left\langle\nabla \Phi\left(u_{n}\right), u_{n}-u\right\rangle \leq 0$, then $\nabla \Phi\left(u_{n}\right) \stackrel{w}{\longrightarrow} \nabla \Phi(u)$ in $\mathfrak{X}^{*}$ and $\Phi\left(u_{n}\right) \rightarrow \Phi(u)$ as $n \rightarrow \infty$.

Proof. From $\left(\Phi_{2}\right)$ and our assumption it follows that

$$
0 \geq \limsup _{n \rightarrow \infty}\left\langle\nabla \Phi\left(u_{n}\right), u_{n}-u\right\rangle \geq \limsup _{n \rightarrow \infty} \Phi\left(u_{n}\right)-\Phi(u) .
$$

On account of $\left(\Phi_{1}\right)$ we obtain

$$
\lim _{n \rightarrow \infty} \Phi\left(u_{n}\right)=\Phi(u)
$$

Next, by $\left(\Phi_{3}\right), \quad \liminf _{n \rightarrow \infty}\left\langle\nabla \Phi\left(u_{n}\right), n_{n}-u\right\rangle \geq \lim _{n \rightarrow \infty}\left\langle\nabla \Phi(u), u_{n}-u\right\rangle=0$. Hence

$$
\lim _{n \rightarrow \infty}\left\langle\nabla \Phi\left(u_{n}\right), u_{n}-u\right\rangle=0
$$

The sequence $\left\{\nabla \Phi\left(u_{n}\right)\right\}$ is weakly relatively compact in $\mathfrak{X}^{*}$, since it is bounded in $\mathfrak{X}^{*}$ on account of $\left(\Phi_{4}\right)$. Now, let $\left\{u_{n_{j}}\right\}$ be any subsequence of $\left\{u_{n}\right\}$ such that $\nabla \Phi\left(u_{n_{j}}\right) \stackrel{w}{\longrightarrow} u^{*}$ in $\mathfrak{X}^{*}$ as $j \rightarrow \infty$ for some $u^{*} \in \mathfrak{X}^{*}$. Then, using $\left(\Phi_{2}\right)$, (1.2) and (1.3), we see that for any $v \in \mathfrak{X}$ 


$$
\begin{aligned}
\left\langle u^{*}, v-u\right\rangle & =\lim _{j \rightarrow \infty}\left\langle\nabla \Phi\left(u_{n_{j}}\right), v-u_{n_{j}}\right\rangle \\
& \leq \lim _{j \rightarrow \infty}\left\{\Phi(v)-\Phi\left(u_{n_{j}}\right)\right\} \\
& =\Phi(v)-\Phi(u),
\end{aligned}
$$

which implies that $u^{*}=\nabla \Phi(u)$, and simultaneously that $\nabla \Phi\left(u_{n}\right) \stackrel{w}{\longrightarrow} \nabla \Phi(u)$ in $\mathfrak{X}^{*}$ as $n \rightarrow \infty$.

q.e.d,

DEFINITION 1.1. (cf. [7], [8]) $\mathfrak{X}=\mathfrak{X}(X ; \xi)$ is called a functional space if the following axiom is satisfied:

AxIom (a) For each compact subset $K$ of $X$, there is a constant $M(K)>0$ such that

$$
\int_{K}|u| d \xi \leq M(K)\|u\| \quad \text { for all } u \in \mathfrak{X} \text {. }
$$

Henceforth let $\mathfrak{X}$ be a functional space satisfying the following axiom:

Axiom $\left(b^{\prime}\right) \quad L^{2} \cap \mathfrak{X}$ is dense both in $L^{2}$ and in $\mathfrak{X}$, where $L^{2}=L^{2}(X ; \xi)$.

We consider an operator $A$ from a subset of $L^{2}$ into $L^{2}$ associated with $\nabla \Phi$ which is defined as follows: If $v$ is a function in $L^{2} \cap \mathfrak{X}$ and $f$ is a function in $L^{2}$ such that

$$
\langle\nabla \Phi(v), w\rangle=\int_{x} w f d \xi \quad \text { for any } w \in L^{2} \cap \mathfrak{X},
$$

then we put $A v=f$. By Axiom ( $\left.\mathrm{b}^{\prime}\right)$, such an $f$ is uniquely determined by $v$. Thus we can define an operator $A: D_{0} \rightarrow L^{2}$, where $D_{0}=\left\{v \in L^{2} \cap \mathfrak{X}\right.$; there is a function $f \in L^{2}$ such that (1.4) holds\}. By $\left(\Phi_{3}\right), A$ is strictly monotone, i.e.,

$$
(A u-A v, u-v)>0 \quad \text { for any } u, v \in D_{0}, u \neq v \text {, }
$$

where $(\cdot, \cdot)$ denotes the inner product in $L^{2}$, i.e., $(v, w)=\int_{X} v w d \xi$ for any $v, w \in L^{2}$.

\section{§ 2. The resolvent of $\mathbf{A}$}

In order to show that the resolvent of $A$ exists, we prove

LEMMA 2.1. Given $\lambda>0$ and $f \in L^{2}$, we find a unique function $u \in L^{2} \cap \mathfrak{X}$ such that 


$$
2 \lambda \Phi(u)+\|u-f\|_{2}^{2}=\inf \left\{2 \lambda \Phi(v)+\|v-f\|_{2}^{2} ; v \in L^{2} \cap \mathfrak{X}\right\},
$$

where by $\|v\|_{2}$ we mean the norm of $v$ in $L^{2}$.

Proof. Set $F(v)=2 \lambda \Phi(v)+\|v-f\|_{2}^{2}$ for $v \in L^{2} \cap \mathfrak{X}$ and $\alpha=\inf \{F(v) ;$ $\left.v \in L^{2} \cap \mathfrak{X}\right\}$. Then, clearly, $0 \leq \alpha<\infty$. We find a sequence $\left\{v_{n}\right\} \subset L^{2} \cap \mathfrak{X}$ such that $F\left(v_{n}\right) \downarrow \alpha$ as $n \rightarrow \infty$. Since $\left\{v_{n}\right\}$ is bounded in $\mathfrak{X}$ by (1.1) as well as $L^{2}$, it is weakly relatively compact both in $\mathfrak{X}$ and in $L^{2}$ and hence, by Axiom (a), there exists a subsequence $\left\{v_{n_{j}}\right\}$ of $\left\{v_{n}\right\}$ such that $v_{n_{j}} \stackrel{w}{\longrightarrow} u$ both in $\mathfrak{X}$ and in $L^{2}$ for some $u \in L^{2} \cap \mathfrak{X}$. Noting $\left(\Phi_{1}\right)$ and the weak lower semicontinuity of the functional: $v \rightarrow\|v-f\|_{2}^{2}$ relative to the topology of $L^{2}$, we have

$$
\Phi(u) \leq \liminf _{j \rightarrow \infty} \Phi\left(v_{n_{j}}\right)
$$

and

$$
\|u-f\|_{2}^{2} \leq \liminf _{j \rightarrow \infty}\left\|v_{n_{j}}-f\right\|_{2}^{2},
$$

so that $F(u) \leq \lim _{j \rightarrow \infty} F\left(v_{n_{j}}\right)=\alpha$. This implies that $F(u)=\alpha$. The uniqueness of such a $u$ follows from the strict convexity of $\Phi$. q.e.d.

For any $\lambda>0$, we denote by $R_{\lambda}$ the operator from $L^{2}$ into $L^{2}$ which assigns the function $u$ satisfying (2.1) to each $f \in L^{2}$.

LEMMA 2.2. Let $\lambda$ be any positive number. Then:

(i) $\Phi\left(R_{\lambda} f\right) \leq \Phi(f)$ for any $f \in L^{2} \cap \mathfrak{X}$.

(ii) $\left\langle\nabla \Phi\left(R_{\lambda} f\right), v\right\rangle=\left(f-R_{\lambda} f, v\right) / \lambda$ for any $f \in L^{2}$ and $v \in L^{2} \cap \mathfrak{X}$.

(iii) $R_{\lambda} f \in D_{0}$ and $A R_{\lambda} f=\left(f-R_{\lambda} f\right) / \lambda$ for any $f \in L^{2}$.

(iv) $R(I+\lambda A$ ) (the range of $I+\lambda A)=L^{2}$ and $R_{\lambda}=(I+\lambda A)^{-1}$.

(v) $\left\|R_{\lambda} f-R_{\lambda} g\right\|_{2} \leq\|f-g\|_{2}$ for any $f, g \in L^{2}$, especially $\left\|R_{\lambda} f\right\|_{2} \leq$ $\|f\|_{2}$ for any $f \in L^{2}$.

Proof. (i) is clear. For any $f \in L^{2}, v \in L^{2} \cap \mathfrak{X}$ and $t>0$, setting $u=R_{\lambda} f$, we observe that

$$
2 \lambda \Phi(u+t v)+\|u+t v-f\|_{2}^{2} \geq 2 \lambda \Phi(u)+\|u-f\|_{2}^{2},
$$

that is

$$
\frac{1}{t}\{\Phi(u+t v)-\Phi(u)\} \geq \frac{1}{2 \lambda} \frac{1}{t}\left\{\|u-f\|_{2}^{2}-\|u+t v-f\|_{2}^{2}\right\} .
$$

Letting $t \downarrow 0$ in this inequality, we obtain 


$$
\langle\nabla \Phi(u), v\rangle \geq \frac{1}{\lambda}(f-u, v)
$$

Hence we have (ii) and at the same time (iii). Besides, we see that

$$
f=R_{\lambda} f+\lambda A R_{\lambda} f=(I+\lambda A) R_{\lambda} f \quad \text { for any } f \in L^{2} .
$$

From this we infer (iv), since $(I+\lambda A)$ is one to one by (1.5). Finally we shall show (v). Let $f$ and $g$ be any functions in $L^{2}$, and set $u=R_{\lambda} f$ and $v=R_{\lambda} g$. Then, as was seen above, $f=u+\lambda A u$ and $g=v+\lambda A v$. From (1.5) it follows that

$$
\begin{aligned}
\|f-g\|_{2}\|u-v\|_{2} & \geq(f-g, u-v) \\
& =(u+\lambda A u-v-\lambda A v, u-v) \\
& =\|u-v\|_{2}^{2}+\lambda(A u-A v, u-v) \\
& \geq\|u-v\|_{2}^{2},
\end{aligned}
$$

so we have (v).

The fact (iv) of Lemma 2.2 says that $R_{\lambda}$ is the (nonlinear) resolvent (at $\lambda$ ) of $A$ for each $\lambda>0$.

LEMMA 2.3 (cf. [9; Lemma 4.3]). If $v \in D_{0}$, then $\left\|A R_{\lambda} v\right\|_{2} \leq\|A v\|_{2}$ for any $\lambda>0$.

Proof. We have $v=R_{\lambda}(I+\lambda A) v=R_{\lambda}(v+\lambda A v)$, since $R_{\lambda}=(I+\lambda A)^{-1}$ by (iv) of Lemma 2.2. From (iii) and (v) of Lemma 2.2 it follows that

$$
\left\|A R_{\lambda} v\right\|_{2}=\frac{1}{\lambda}\left\|v-R_{\lambda} v\right\|_{2}=\frac{1}{\lambda}\left\|R_{\lambda}(v+\lambda A v)-R_{\lambda} v\right\|_{2} \leq\|A v\|_{2} \text {. } \quad \text { q.e.d. }
$$

LEMMA 2.4. (1) For any $f \in L^{2}, R_{\lambda} f \stackrel{s}{\longrightarrow} f$ in $L^{2}$ as $\lambda \downarrow 0$.

(2) For any $f \in L^{2} \cap \mathfrak{X}, R_{\lambda} f \stackrel{w}{\longrightarrow} f$ in $\mathfrak{X}$ as $\lambda \downarrow 0$.

Proof. First, let $f$ be any function in $L^{2} \cap \mathfrak{X}$. We observe from (1.1) and (i), (v) of Lemma 2.2 that

$$
C\left\|R_{\lambda} f\right\|^{p} \leq \Phi\left(R_{\lambda} f\right) \leq \Phi(f) \quad \text { for any } \lambda>0
$$

and

$$
\left\|R_{\lambda} f\right\|_{2} \leq\|f\|_{2} \quad \text { for any } \lambda>0 .
$$

Therefore $\left\{R_{\lambda} f ; \lambda>0\right\}$ is bounded in $\mathfrak{X}$ as well as in $L^{2}$. From (ii) of Lemma 2.2 and $\left(\Phi_{4}\right)$ we derive that for each $v \in L^{2} \cap \mathfrak{X}$ 


$$
\left(f-R_{\lambda} f, v\right)=\lambda\left\langle\nabla \Phi\left(R_{\lambda} f\right), v\right\rangle \rightarrow 0 \quad \text { as } \lambda \downarrow 0 .
$$

This fact and the boundedness of $\left\{R_{\lambda} f ; \lambda>0\right\}$ in $L^{2}$ imply that

$$
R_{\lambda} f \stackrel{w}{\longrightarrow} f \quad \text { in } L^{2} \text { as } \lambda \downarrow 0
$$

and $\liminf _{\lambda \downarrow 0}\left\|R_{\lambda} f\right\|_{2} \geq\|f\|_{2}$. On the other hand, we have by (2.2) $\limsup _{\lambda \downarrow 0}\left\|R_{\lambda} f\right\|_{2} \leq\|f\|_{2}$. Hence, $\lim _{\lambda \downarrow 0}\left\|R_{\lambda} f\right\|_{2}=\|f\|_{2}$. This together with (2.3) implies that

$$
R_{\lambda} f \stackrel{s}{\longrightarrow} f \quad \text { in } L^{2} \text { as } \lambda \downarrow 0 \text {. }
$$

Now, let $\left\{\lambda_{j}\right\}$ be any sequence tending to 0 such that $\left\{R_{\lambda_{j}} f\right\}$ is weakly convergent in $\mathfrak{X}$. Then, denoting the weak limit by $g$, we see from (2.4) and Axiom (a) that $g=f$. This shows that $R_{\lambda} f \stackrel{w}{\longrightarrow} f$ in $\mathfrak{X}$ as $\lambda \downarrow 0$. Finally we can obtain (1) by using Axiom (b') and (v) of Lemma 2.2 and noting the fact that $R_{\lambda} f \stackrel{s}{\longrightarrow} f$ in $L^{2}$ as $\lambda \downarrow 0$ for $f \in L^{2} \cap \mathfrak{X}$.

q.e.d.

Remark. By the above lemma, $D_{0}$ is dense in $L^{2}$. Also, we can prove that $D_{0}$ is weakly sequentially dense in $\mathfrak{X}$.

LEMMA 2.5. If $f \in L^{2} \cap \mathfrak{X}$, then $\nabla \Phi\left(R_{\lambda} f\right) \stackrel{w}{\longrightarrow} \nabla \Phi(f)$ in $\mathfrak{X}^{*}$ and $\Phi\left(R_{\lambda} f\right) \rightarrow \Phi(f)$ as $\lambda \downarrow 0$.

Proof. Let $f \in L^{2} \cap \mathfrak{X}$. Then we see from (ii) of Lemma 2.2 that

$$
\underset{\lambda \downarrow 0}{\limsup }\left\langle\nabla \Phi\left(R_{\lambda} f\right), R_{\lambda} f-f\right\rangle=\limsup _{\lambda \downarrow 0}-\frac{1}{\lambda}\left\|f-R_{\lambda} f\right\|_{2}^{2} \leq 0 .
$$

Applying Lemma 1.1, we obtain the lemma.

q.e.d.

Following Deny (cf. [7; Théorème 2]), we define

$$
H_{\lambda}(f)=\frac{1}{\lambda}\left(f-R_{\lambda} f, f\right), \quad f \in L^{2} .
$$

We note that the following relations hold:

$$
H_{\lambda}(f) \geq \frac{1}{\lambda}\left(f-R_{\lambda} f, R_{\lambda} f\right)=\left\langle\nabla \Phi\left(R_{\lambda} f\right), R_{\lambda} f\right\rangle \geq \Phi\left(R_{\lambda} f\right) .
$$

LEMMA 2.6. If $f \in L^{2}$ and if $\left\{H_{\lambda}(f) ; \lambda>0\right\}$ is bounded, then $f \in \mathfrak{X}$.

Proof. Let $f \in L^{2}$ and assume that $\left\{H_{\lambda}(f) ; \lambda>0\right\}$ is bounded. Then 
we see from (2.5) and (1.1) that $\left\{R_{\lambda} f ; \lambda>0\right\}$ is bounded in $\mathfrak{X}$. Hence there is a sequence $\left\{\lambda_{j}\right\}$ tending to 0 such that $R_{\lambda_{j}} f \stackrel{w}{\longrightarrow} g$ in $\mathfrak{X}$ for some $g \in \mathfrak{X}$. By (1) of Lemma 2.4 and Axiom (a), we have $g=f$, so that $f \in \mathfrak{X}$.

\section{§3. Potential theoretic properties and their equivalence}

In this section, we state potential theoretic properties of $\Phi, A$ and $R_{\lambda}$ and our main results about their equivalence.

Definition 3.1 (cf. [5], [11]). Let $k$ be a positive number or $\infty$. We say that the contraction $T_{k}$ operates in $\mathfrak{X}$ with respect to $\Phi$ if the following two conditions are satisfied:

$\left(C_{k}\right) \quad T_{k} v \equiv v^{+} \wedge k \in \mathfrak{X} \quad$ for all $v \in \mathfrak{X}$, where $T_{k} v=v^{+}$if $k=\infty$.

$\left(\Phi C_{k}\right)$ For any $u, v \in \mathfrak{X}$,

$$
\Phi\left(u+T_{k}(v-u)\right)+\Phi\left(v-T_{k}(v-u)\right) \leq \Phi(u)+\Phi(v) .
$$

In particular, we say that the modulus contraction operates in $\mathfrak{X}$ with respect to $\Phi$, when $T_{\infty}$ operates in $\mathfrak{X}$ with respect to $\Phi$.

Remark. It was shown in [11; Proposition 2.1] that under $\left(C_{k}\right)$, condition $\left(\Phi C_{k}\right)$ is equivalent to the following:

$$
\left(\Phi C_{k}\right)^{\prime}\left\langle\nabla \Phi\left(u+T_{k} v\right)-\nabla \Phi(u), v-T_{k} v\right) \geq 0 \quad \text { for any } u, v \in \mathfrak{X} .
$$

In particular, $\left(\Phi C_{\infty}\right)^{\prime}$ is of the form:

$$
\left\langle\nabla \Phi\left(u+v^{+}\right)-\nabla \Phi(u), v^{-}\right\rangle \leq 0 \quad \text { for any } u, v \in \mathfrak{X} .
$$

DEFINITION 3.2 (cf. [5], [11]). We say that the principle of lower envelope with respect to $\Phi$ holds if $\left(C_{\infty}\right)$ and the following are satisfied:

$(\Phi L)$ If $u$ and $v$ are functions in $D_{0}$, then

$$
\langle\nabla \Phi(u \wedge v), w\rangle \geq(A u \wedge A v, w)
$$

for any non-negative function $w \in L^{2} \cap \mathfrak{X}$.

DEFINITION 3.3 (cf. [5], [11]). We say that the domination principle with respect to $\Phi$ holds, if the following is satisfied:

(ФD) If $u$ and $v$ are functions in $D_{0}$ and if there is $f \in L^{2}$ such that $A u \geq f, A v \geq f$ and $\left(A u-f,(u-v)^{+}\right)=0$, then $u \leq v$.

Definition 3.4 (cf. [5], [11]). We say that $R_{\lambda}, \lambda>0$, is orderpreserving in $L^{2}$, if the following holds :

(ФО) $R_{\lambda} f \leq R_{\lambda} g$ for any $f, g \in L^{2}$ such that $f \leq g$. 
Now, we are in a position to state one of main theorems.

THEOREM A. The following statements are equivalent to each other:

$\left(a_{1}\right)$ The modulus contraction operates in $\mathfrak{X}$ with respect to $\Phi$.

$\left(a_{2}\right)$ The principle of lower envelope with respect to $\Phi$ holds.

$\left(a_{3}\right)$ The domination principle with respect to $\Phi$ holds.

$\left(a_{4}\right)$ For any $\lambda>0$, the resolvent $R_{\lambda}$ is order-preserving in $L^{2}$.

Next, to state another main theorem we give some definitions. Throughout the remainder of this section, let $k$ be a positive number.

Definition 3.5 (cf. [5], [11]). We say that the strong principle of lower envelope with respect to $\Phi$ and $k$ holds, if the following two conditions are satisfied:

$\left(C_{k}\right)^{*} \quad u \wedge(v+k) \in \mathfrak{X} \quad$ for any $u, v \in \mathfrak{X}$.

$\left(\Phi S L_{k}\right)$ If $u$ and $v$ are functions in $D_{0}$, then

$$
\langle\nabla \Phi(u \wedge(v+k)), w\rangle \geq(A u \wedge A v, w)
$$

for any non-negative function $w \in L^{2} \cap \mathfrak{X}$.

It should be noticed that under $\left(C_{\infty}\right)$, conditions $\left(C_{k}\right)^{*}$ and $\left(C_{k}\right)$ are equivalent.

DEFINITION 3.6 (cf. [5], [11]). We say that the complete maximum principle with respect to $\Phi$ and $k$ holds, if the following is satisfied:

$\left(\Phi C M_{k}\right)$ If $u$ and $v$ are functions in $D_{0}$ and if there is $f \in L^{2}$ such that $A u \geq f, A v \geq f$ and $\left(A u-f,(u-v-k)^{+}\right)=0$, then $u \leq v+k$.

The second main theorem is stated as follows:

THEOREM B. Assume that the modulus contraction operates in $\mathfrak{X}$ with respect to $\Phi$. Then the following statements are equivalent to each other:

$\left(b_{1}\right)$ The contraction $T_{k}$ operates in $\mathfrak{X}$ with respect to $\Phi$.

$\left(b_{2}\right)$ The strong principle of lower envelope with respect to $\Phi$ and $k$ holds.

$\left(b_{3}\right)$ The complete maximum principle with respect to $\Phi$ and $k$ holds.

$\left(b_{4}\right)$ For any $\lambda>0$ and any $f, g \in L^{2}$

$$
R_{\lambda}\left(f+T_{k} g\right) \leq R_{\lambda} f+k .
$$

\section{§ 4. Proofs of Theorems $A$ and $B$}

Before proving the theorems, we recall an existence theorem for 
nonlinear variational inequalities. Let $\mathfrak{Y}$ be a real reflexive Banach space and $P$ be a (nonlinear) semicontinuous monotone ${ }^{1)}$ operator from $\mathfrak{Y}$ into $\mathfrak{Y}^{*}$ (the dual space of $\mathfrak{Y}$ ). Let $\Psi$ be a lower semicontinuous convex function on $\mathfrak{V}$ with values in $(-\infty, \infty]$.

THEOREM (cf. [3; Theorem 3], [10; Theorem 4.1]). Let $\Re$ be a nonempty closed convex subset of $\mathfrak{V}$ and assume that for some $w \in \Re$ with $\Psi(w)<\infty$,

$$
\frac{\langle P v, v-w\rangle_{\vartheta}+\Psi(v)}{\|v\|_{\eta}} \rightarrow \infty \quad \text { as }\|v\|_{\mathscr{\eta}} \rightarrow \infty, v \in \Re,
$$

where we denote by $\langle\cdot, \cdot\rangle_{\mathfrak{g}}$ the natural pairing between $\mathfrak{Y} *$ and $\mathfrak{Y}$ and by $\|\cdot\|_{\mathfrak{g}}$ the norm in $\mathfrak{Y}$. Then, there is $u \in \Re$ such that

$$
\langle P u, u-v\rangle_{\mathfrak{g}} \leq \Psi(v)-\Psi(u) \quad \text { for all } v \in \Re .
$$

Moreover, if $\boldsymbol{P}$ is strictly monotone, then such $a u$ is unique.

LEMMA 4.1. The function $\Psi$ on $L^{2}$ defined by

$$
\Psi(v)= \begin{cases}\Phi(v), & \text { if } v \in L^{2} \cap \mathfrak{X}, \\ \infty, & \text { otherwise, }\end{cases}
$$

is lower semicontinuous on $L^{2}$.

Proof. Let $\left\{v_{n}\right\}$ be a sequence in $L^{2}$ which converges to $v_{0}$ in $L^{2}$, and assume that $\alpha \equiv \liminf _{n \rightarrow \infty} \Psi\left(v_{n}\right)<\infty$. Then, by (1.1) there is a subsequence $\left\{v_{n_{j}}\right\}$ of $\left\{v_{n}\right\}$ such that $v_{n_{j}} \stackrel{w}{\longrightarrow} v$ in $\mathfrak{X}$ for some $v \in \mathfrak{X}$ and $\Phi\left(v_{n_{j}}\right) \rightarrow \alpha$ as $j \rightarrow \infty$. By Axiom (a), $v_{0}=v \in \mathfrak{X}$. Hence, from $\left(\Phi_{1}\right)$ it follows that $\alpha \geq \Phi\left(v_{0}\right)=\Psi\left(v_{0}\right)$. Thus $\Psi$ is lower semicontinuous on $L^{2}$.

q.e.d.

Proof of $\left(a_{1}\right) \rightarrow\left(a_{2}\right)$ : Let $u \in D_{0}$ and $v \in D_{0}$, and set $f=A u$ and $g=A v$. Define an operator $P: L^{2} \rightarrow L^{2}$ by $P w=w-u \wedge v-f \wedge g$ and let $\Psi$ be the same function as in Lemma 4.1. Obviously, $P$ is a demicontinuous monotone operator from $L^{2}$ into $L^{2}$ and $\Psi$ is a convex function on $L^{2}$ with values in $[0, \infty]$ such that $\{(P w, w-u)+\Psi(w)\} /\|w\|_{2} \rightarrow \infty$ as $\|w\|_{2} \rightarrow \infty$. By Lemma 4.1, $\Psi$ is lower semicontinuous on $L^{2}$. Furthermore, setting $\AA=\left\{w \in L^{2} ; w \geq u \wedge v\right\}$, we see that $\AA$ is closed and convex in $L^{2}, u \in \Re$ and $\Psi(u)=\Phi(u)<\infty$. By virtue of the existence theorem

1) An operator $P$ from $\mathfrak{V}$ into $\mathfrak{Y}^{*}$ is called monotone, if for any $u, v \in \mathfrak{V},\langle P u-P v$, $u-v\rangle_{\vartheta} \geq 0$. 
mentioned above, there is $u_{0} \in \Re$ such that $\left(P u_{0}, u_{0}-w\right) \leq \Psi(w)-\Psi\left(u_{0}\right)$ for all $w \in \Re$. Clearly, $u_{0} \in \mathfrak{\Re} \cap \mathfrak{X}$, so we have $\left(P u_{0}, u_{0}-w\right) \leq \Phi(w)-\Phi\left(u_{0}\right)$ for all $w \in \Re \cap \mathfrak{X}$. Since $u_{0}+t\left(w-u_{0}\right) \in \Re \cap \mathfrak{X}$ for any $w \in \Re \cap \mathfrak{X}$ and $1>t>0$, we see that

$$
\left(P u_{0}, u_{0}-w\right) \leq \frac{1}{t}\left\{\Phi\left(u_{0}+t\left(w-u_{0}\right)\right)-\Phi\left(u_{0}\right)\right\} .
$$

As $t \downarrow 0$, we have

$$
\left(P u_{0}, u_{0}-w\right) \leq\left\langle\nabla \Phi\left(u_{0}\right), w-u_{0}\right\rangle \quad \text { for any } w \in \mathfrak{\Re} \cap \mathfrak{X} .
$$

By $\left(a_{1}\right), u_{0} \wedge u \in \Re \cap \mathfrak{X}$. Hence, taking $w=u_{0} \wedge u$ in (4.1)

$$
\left(P u_{0}, u_{0}-u \wedge u_{0}\right) \leq\left\langle\nabla \Phi\left(u_{0}\right), u \wedge u_{0}-u_{0}\right\rangle .
$$

Since $f+P u=u-u \wedge v+f-f \wedge g \geq 0$, it follows from $\left(\Phi C_{\infty}\right)^{\prime}$ that $\left\langle\nabla \Phi\left(u \wedge u_{0}\right), u_{0}-u \wedge u_{0}\right\rangle=\left\langle\nabla \Phi\left(u-\left(u-u_{0}\right)^{+}\right),\left(u-u_{0}\right)^{-}\right\rangle \geq\langle\nabla \Phi(u),(u-$ $\left.\left.u_{0}\right)^{-}\right\rangle=\left(f, u_{0}-u \wedge u_{0}\right) \geq\left(-P u, u_{0}-u \wedge u_{0}\right)$. Hence, by (4.2), $\left\langle\nabla \Phi\left(u_{0}\right)-\right.$ $\left.\nabla \Phi\left(u \wedge u_{0}\right), u_{0}-u \wedge u_{0}\right\rangle \leq-\left(P u_{0}-P u,\left(u_{0}-u\right)^{+}\right) \leq 0$, so we have by $\left(\Phi_{3}\right)$ $u_{0}=u_{0} \wedge u$, that is, $u_{0} \leq u$. In a similar manner, we have $u_{0} \leq v$. Therefore $u_{0} \leq u \wedge v$, while $u_{0} \geq u \wedge v$ because $u_{0} \in \Re$. Consequently, $u_{0}=u \wedge v$ and hence (4.1) yields $(\Phi L)$.

q.e.d.

Proof of $\left\{\left(a_{1}\right),\left(b_{1}\right)\right\} \rightarrow\left(b_{2}\right)$ : Let $u$ and $v$ be any functions in $D_{0}$, and set $f=A u$ and $g=A v$. Define an operator $P$ from $L^{2}$ into $L^{2}$ by $P w=w-u \wedge(v+k)-f \wedge g$ and denote by $\Re$ the set of all $w \in L^{2}$ such that $w \geq u \wedge(v+k)$. Then, in the same manner as in the proof of $\left(a_{1}\right) \rightarrow\left(a_{2}\right)$, we can find $u_{0} \in \Re \cap \mathfrak{X}$ such that $u_{0} \leq u$ and

$$
\left(P u_{0}, u_{0}-w\right) \leq\left\langle\nabla \Phi\left(u_{0}\right), w-u_{0}\right\rangle \quad \text { for any } w \in \Re \cap \mathfrak{X} .
$$

Moreover, $u_{0}=u_{0} \wedge(v+k)$ holds. In fact, by using $\left(\Phi C_{k}\right)^{\prime}$ we observe that

$$
\begin{aligned}
\langle\nabla \Phi & \left.\left(u_{0} \wedge(v+k)\right), u_{0}-u_{0} \wedge(v+k)\right\rangle \\
& =\left\langle\nabla \Phi\left(u_{0} \wedge v+T_{k}\left(\left(u_{0}-v\right)^{+}\right)\right),\left(u_{0}-v\right)^{+}-T_{k}\left(\left(u_{0}-v\right)^{+}\right)\right\rangle \\
& \geq\left\langle\nabla \Phi\left(u_{0} \wedge v\right),\left(u_{0}-v\right)^{+}-T_{k}\left(\left(u_{0}-v\right)^{+}\right)\right\rangle \\
& =\left\langle\nabla \Phi\left(v-\left(v-u_{0}\right)^{+}\right),\left(u_{0}-v-k\right)^{+}\right\rangle .
\end{aligned}
$$

Now, putting $z=\left(v-u_{0}\right)^{+}-\left(u_{0}-v-k\right)^{+}$, we see that $w^{+}=\left(v-u_{0}\right)^{+}$ and $w^{-}=\left(u_{0}-v-k\right)^{+}$, so by $\left(\Phi C_{\infty}\right)^{\prime}$ the right hand side of the above inequalities is 


$$
\geq\left\langle\nabla \Phi\left(v-\left(v-u_{0}\right)^{+}+w^{+}\right), w^{-}\right\rangle=\left(g, u_{0}-u_{0} \wedge(v+k)\right) .
$$

From this and (4.3) it follows that

$$
\begin{gathered}
\left\langle\nabla \Phi\left(u_{0}\right)-\nabla \Phi\left(u_{0} \wedge(v+k)\right), u_{0}-u_{0} \wedge(v+k)\right\rangle \\
\leq-\left(P u_{0}+g, u_{0}-u_{0} \wedge(v+k)\right) \leq 0 .
\end{gathered}
$$

By $\left(\Phi_{3}\right), u_{0}=u_{0} \wedge(v+k)$. Hence we see that $u_{0}=u \wedge(v+k)$ and obtain $\left(\Phi S L_{k}\right)$ from (4.3). $\quad$ q.e.d.

Proof of $\left(b_{2}\right) \rightarrow\left(b_{3}\right)$ : Let $u$ and $v$ be any functions in $D_{0}$ and $f$ be a function in $L^{2}$ such that $A u \geq f, A v \geq f$ and $\left(A u-f,(u-v-k)^{+}\right)=0$. Then it follows from $\left(\Phi S L_{k}\right)$ that

$\langle\nabla \Phi(u \wedge(v+k)), w\rangle \geq(f, w) \quad$ for any non-negative $w \in L^{2} \cap \mathfrak{X}$.

Hence

$$
\begin{aligned}
& \langle\nabla \Phi(u)-\nabla \Phi(u \wedge(v+k)), u-u \wedge(v+k)\rangle \\
& \quad=\left\langle\nabla \Phi(u)-\nabla \Phi(u \wedge(v+k)),(u-v-k)^{+}\right\rangle \\
& \quad \leq\left(A u-f,(u-v-k)^{+}\right) \\
& \quad=0 .
\end{aligned}
$$

From this and $\left(\Phi_{3}\right)$ we obtain $u=u \wedge(v+k)$, that is, $u \leq v+k$.

Proof of $\left(a_{2}\right) \rightarrow\left(a_{3}\right)$ : By taking 0 instead of $k$ in the above proof, we have the proof of $\left(a_{2}\right) \rightarrow\left(a_{3}\right)$.

Proof of $\left(b_{3}\right) \rightarrow\left(b_{4}\right)$ : Let $\lambda$ be any positive number and $f$ and $g$ be any functions in $L^{2}$. Set $u=R_{\lambda}\left(f+T_{k} g\right)$ and $v=R_{\lambda} f$. Then we see by (iii) of Lemma 2.2 that $A u \geq \lambda^{-1}\left\{f-\left(u-T_{k} g\right) \vee v\right\}$ and $A v \geq$ $\lambda^{-1}\left\{f-\left(u-T_{k} g\right) \vee v\right\}$. Moreover,

$$
\left(A u-\frac{f-\left(u-T_{k} g\right) \vee v}{\lambda},(u-v-k)^{+}\right)=0 \text {. }
$$

Hence, we obtain from $\left(\Phi C M_{k}\right)$ that $u \leq v+k$, i.e., $R_{\lambda}\left(f+T_{k} g\right) \leq R_{\lambda} f+k$. q.e.d.

Proof of $\left(a_{3}\right) \rightarrow\left(a_{4}\right):$ In the above proof, replace $k$ by 0 and $T_{k} g$ by $-g^{+}$. Then, by $(\Phi D)$ we have $R_{\lambda}\left(f-g^{+}\right) \leq R_{\lambda} f$. Since this inequality holds for any $f, g \in L^{2}, R_{\lambda}$ is order-preserving in $L^{2}$.

Remark. The proofs of $\left(a_{2}\right) \rightarrow\left(a_{3}\right) \rightarrow\left(a_{4}\right)$ and of $\left(b_{2}\right) \rightarrow\left(b_{3}\right) \rightarrow\left(b_{4}\right)$ given above are essentially due to Calvert $[5 ; \S 2]$. 
In order to prove the assertions $\left(a_{4}\right) \rightarrow\left(a_{1}\right)$ and $\left\{\left(a_{4}\right),\left(b_{4}\right)\right\} \rightarrow\left(b_{1}\right)$, we prepare some lemmas. In the rest of this section, let $k$ be a positive number.

LEMMA 4.2. (i) If $\left(a_{4}\right)$ and $\left(b_{4}\right)$ are satisfied, then $T_{k} f \in \mathfrak{X}$ for any $f \in D_{0}$.

(ii) If $\left(a_{4}\right)$ is satisfied, then $f^{+} \in \mathfrak{X}$ for any $f \in D_{0}$.

Proof. Assume $\left(a_{4}\right)$ and $\left(b_{4}\right)$. Let $f \in D_{0}$ and set $g=T_{k} f$. Then

$$
\begin{aligned}
H_{\lambda}(f-g) & =\frac{1}{\lambda}\left((f-g)-R_{\lambda}(f-g), f-g\right) \\
& =\frac{1}{\lambda} \sum_{i=1}^{3} \int_{X_{i}}\left\{(f-g)-R_{\lambda}(f-g)\right\}(f-g) d \xi,
\end{aligned}
$$

where $X_{1}=\{x \in X ; f(x)<0\}, X_{2}=\{x \in X ; 0 \leq f(x) \leq k\}$ and $X_{3}=\{x \in X$; $f(x)>k\}$. By $\left(a_{4}\right)$ and $\left(b_{4}\right)$ we have

$$
R_{\lambda}(f-g) \leq R_{\lambda} f \leq R_{\lambda}(f-g)+k,
$$

so that $(f-g)-R_{\lambda}(f-g) \geq f-R_{\lambda} f$ on $X_{1}$ and $\leq f-R_{\lambda} f$ on $X_{3}$. Moreover, $f-g=f<0$ on $X_{1}, f-g=0$ on $X_{2}$ and $0<f-g<f$ on $X_{3}$. Hence we have $H_{\lambda}(f-g) \leq \lambda^{-1}\left\|f-R_{\lambda} f\right\|_{2}\|f\|_{2} \leq\|A f\|_{2}\|f\|_{2}$ because of (iii) of Lemma 2.2 and Lemma 2.3, so from Lemma 2.6 it follows that $f-g \in \mathfrak{X}$, i.e., $g \in \mathfrak{X}$. Thus (i) is obtained. (ii) is similarly proved.

q.e.d.

LEMMA 4.3. (i) If $\left(a_{4}\right)$ and $\left(b_{4}\right)$ are satisfied, then

$$
\left\langle\nabla \Phi\left(u+T_{k} v\right)-\nabla \Phi(u), v-T_{k} v\right\rangle \geq 0 \quad \text { for any } u \in \mathfrak{X} \text { and } v \in D_{0} \text {. }
$$

(ii) If $\left(a_{4}\right)$ is satisfied, then

$$
\left\langle\nabla \Phi\left(u+v^{+}\right)-\nabla \Phi(u), v^{-}\right\rangle \leq 0 \quad \text { for any } u \in \mathfrak{X} \text { and } v \in D_{0} .
$$

Proof. Assume $\left(a_{4}\right)$ and $\left(b_{4}\right)$. According to Axiom $\left(b^{\prime}\right)$ and property $\left(\Phi_{5}\right)$, it is sufficient to show the inequality in case $u \in L^{2} \cap \mathfrak{X}$ and $v \in D_{0}$. By Lemma 4.2 we see that $T_{k} v \in L^{2} \cap \mathfrak{X}$. Furthermore we have

$$
\begin{aligned}
& \left\langle\nabla \Phi\left(R_{\lambda}\left(u+T_{k} v\right)\right)-\nabla \Phi\left(R_{\lambda} u\right), v-T_{k} v\right\rangle \\
& \quad=\frac{1}{\lambda}\left(\left(u+T_{k} v\right)-R_{\lambda}\left(u+T_{k} v\right)-\left(u-R_{\lambda} u\right), v-T_{k} v\right) \\
& \quad=\frac{1}{\lambda}\left(T_{k} v+R_{\lambda} u-R_{\lambda}\left(u+T_{k} v\right), v=T_{k} v\right) .
\end{aligned}
$$


Now, $T_{k} v+R_{\lambda} u-R_{\lambda}\left(u+T_{k} v\right) \geq 0$ on $X_{1}=\{x \in X ; v(x) \geq k\}$ by $\left(b_{4}\right)$ and $\leq 0$ on $X_{2}=\{x \in X ; v(x) \leq 0\}$ by $\left(a_{4}\right)$. Moreover, $v-T_{k} v \geq 0$ on $X_{1}$, $\leq 0$ on $X_{2}$ and $=0$ on $\{x \in X ; 0<v(x)<k\}$, so the right hand side of the above equalities is non-negative. Hence, by Lemma 2.5,

$$
\begin{aligned}
& \left\langle\nabla \Phi\left(u+T_{k} v\right)-\nabla \Phi(u), v-T_{k} v\right\rangle \\
& \quad=\lim _{\lambda \downarrow 0}\left\langle\nabla \Phi\left(R_{\lambda}\left(u+T_{k} v\right)\right)-\nabla \Phi\left(R_{\lambda} u\right), u-T_{k} v\right\rangle \\
& \quad \geq 0 .
\end{aligned}
$$

The assertion (ii) is similarly proved.

q.e.d.

LEMMA 4.4. (i) If $\left(a_{4}\right)$ and $\left(b_{4}\right)$ are satisfied, then

$$
\Phi(u)+\Phi(u+v) \geq \Phi\left(u+T_{k} v\right)+\Phi\left(u+v-T_{k} v\right)
$$

for any $u \in \mathfrak{X}$ and $v \in D_{0}$.

(ii) If $\left(a_{4}\right)$ is satisfied, then

$$
\Phi(u)+\Phi(u+v) \geq \Phi\left(u+v^{+}\right)+\Phi\left(u-v^{-}\right)
$$

for any $u \in \mathfrak{X}$ and $v \in D_{0}$.

Proof. We shall show (i). By using property $\left(\Phi_{7}\right)$ and (i) of Lemma 4.3 we have

$$
\begin{aligned}
& \Phi(u+v)-\Phi\left(u+T_{k} v\right) \\
& \quad=\int_{0}^{1}\left\langle\nabla \Phi\left(u+T_{k} v+t\left(v-T_{k} v\right)\right), v-T_{k} v\right\rangle d t \\
& \quad \geq \int_{0}^{1}\left\langle\nabla \Phi\left(u+t\left(v-T_{k} v\right)\right), v-T_{k} v\right\rangle d t \\
& \quad=\Phi\left(u+v-T_{k} v\right)-\Phi(u)
\end{aligned}
$$

for any $u \in \mathfrak{X}$ and $v \in D_{0}$. Similarly from $\left(\Phi_{7}\right)$ and (ii) of Lemma 4.3 we obtain (ii).

q.e.d.

Proof of $\left.\left\{a_{4}\right),\left(b_{4}\right)\right\} \rightarrow\left(b_{1}\right)$ : Let $v$ be any function in $L^{2} \cap \mathfrak{X}$. Then $R_{\lambda} v \in D_{0}, \lambda>0$, because of (iii) of Lemma 2.2. Taking 0 and $R_{\lambda} v$ for $u$ and $v$ in (4.5) respectively and using (i) of Lemma 2.2, we have

$$
\Phi\left(T_{k}\left(R_{\lambda} v\right)\right) \leq \Phi\left(R_{\lambda} v\right) \leq \Phi(v),
$$

so by (1.1), $\left\{T_{k}\left(R_{\lambda} v\right) ; \lambda>0\right\}$ is bounded in $\mathfrak{X}$ and hence it is weakly relatively compact in $\mathfrak{X}$. Now, let $\left\{T_{k}\left(R_{\lambda_{n}} v\right)\right\}$ be any sequence weakly convergent in $\mathfrak{X}$ such that $\lambda_{n} \downarrow 0$ as $n \rightarrow \infty$, and denote by $g$ the weak limit. 
Then, since $T_{k}\left(R_{\lambda_{n}} v\right) \stackrel{s}{\longrightarrow} T_{k} v$ in $L^{2}$ as $n \rightarrow \infty$ by (i) of Lemma 2.4, it follows that $g=T_{k} v$. This shows that $T_{k} v \in \mathfrak{X}$. Thus we have seen that $T_{k} v \in \mathfrak{X}$ for any $v \in L^{2} \cap \mathfrak{X}$. Moreover, just as Lemma 4.3, we can prove that

$$
\left\langle\nabla \Phi\left(u+T_{k} v\right)-\nabla \Phi(u), v-T_{k} v\right\rangle \geq 0 \text { for any } u \in \mathfrak{X} \text { and } v \in L^{2} \cap \mathfrak{X} .
$$

From this, by the same calculation as in the proof of Lemma 4.4, we deduce that

$$
\begin{aligned}
\Phi\left(u+T_{k} v\right)+\Phi\left(u+v-T_{k} v\right) & \leq \Phi(u)+\Phi(u+v) \\
& \text { for any } u \in \mathfrak{X} \text { and } v \in L^{2} \cap \mathfrak{X} .
\end{aligned}
$$

Again by the limit process, we see from (4.6) that $T_{k} v \in \mathfrak{X}$ for any $v \in \mathfrak{X}$, i.e., $\left(C_{k}\right)$ holds, and simultaneously that

$$
\Phi\left(u+T_{k} v\right)+\Phi\left(u+v-T_{k} v\right) \leq \Phi(u)+\Phi(u+v)
$$

for any $u, v \in \mathfrak{X}$. Clearly, this inequality is equivalent to $\left(\Phi C_{k}\right)$. q.e.d.

Proof of $\left(a_{4}\right) \rightarrow\left(a_{1}\right)$ : We can prove the assertion just as $\left\{\left(a_{4}\right),\left(b_{4}\right)\right\}$ $\rightarrow\left(b_{1}\right)$.

\section{§6. The nonlinear contraction semigroup generated by $-A$}

In this section we discuss the nonlinear contraction semigroup on $L^{2}$ generated by $-A$. In view of the generation theorem for contraction semigroups due to Kömura [12; Theorem 4], there is a unique contraction semigroup $S=\{S(t) ; t \geq 0\}$ on $L^{2}$ whose infinitesimal generator is $-A$. Here we mean by a contraction semigroup $S=\{S(t) ; t \geq 0\}$ on $L^{2}$ a one-parameter family of operators $S(t), t \geq 0$, from $L^{2}$ into $L^{2}$ with the following properties:

$\left(s_{1}\right) \quad S(t) S(s)=S(t+s) \quad$ for $t, s \geq 0$.

$\left(s_{2}\right) \quad S(0)=I$.

$\left(s_{3}\right) \quad\|S(t) v-S(t) w\|_{2} \leq\|v-w\|_{2} \quad$ for $t \geq 0$ and $v, w \in L^{2}$.

$\left(s_{4}\right)$ For each $v \in L^{2}, t \rightarrow S(t) v$ is continuous on $[0, \infty)$.

The contraction semigroup $S=\{S(t) ; t \geq 0\}$ on $L^{2}$ generated by $-A$ is in fact given by

$$
S(t) v=\lim _{n \rightarrow \infty} R_{t / n}^{n} v \quad \text { for } t \geq 0 \text { and } v \in L^{2}
$$


(see Crandall-Liggett [6; Theorem I]).

THEOREM C. The following statements $\left(c_{1}\right) \sim\left(c_{3}\right)$ and $\left(a_{1}\right) \sim\left(a_{4}\right)$ (in Theorem $A$ ) are equivalent to each other:

(c) $A$ is T-accretive (or $-A$ is dispersive) (cf. [4], [13]), i.e.,

$$
\left(A u-A v,(u-v)^{+}\right) \geq 0 \quad \text { for any } u, v \in D_{0} .
$$

(c) For any $t, s \in[0, \infty), s \leq t$, and any $u, v \in L^{2}$,

$$
\left\|(S(t) u-S(t) v)^{+}\right\|_{2} \leq\left\|(S(s) u-S(s) v)^{+}\right\|_{2} .
$$

(c) For any $\lambda>0$ and any $u, v \in L^{2}$,

$$
\left\|\left(R_{\lambda} u-R_{\lambda} v\right)^{+}\right\|_{2} \leq\left\|(u-v)^{+}\right\|_{2} .
$$

Proof. From $\left(a_{1}\right)$ we see that

$\left(c_{1}^{\prime}\right)$ Condition $\left(C_{\infty}\right)$ is satisfied and $\nabla \Phi$ is $T$-monotone (cf. [1]), i.e., for any $u, v \in \mathfrak{X}$,

$$
\left\langle\nabla \Phi(u)-\nabla \Phi(v),(u-v)^{+}\right\rangle \geq 0 .
$$

In fact, from $\left(\Phi C_{\infty}\right)^{\prime}$ and the monotonicity of $\nabla \Phi$ we derive that

$$
\begin{aligned}
& \left\langle\nabla \Phi(u)-\nabla \Phi(v),(u-v)^{+}\right\rangle \\
& \quad=\left\langle\nabla \Phi(u)-\nabla \Phi(v),(v-u)^{-}\right\rangle \\
& \quad \geq\left\langle\nabla \Phi\left(u+(v-u)^{+}\right)-\nabla \Phi(v),(v-u)^{-}\right\rangle \\
& \quad \geq\langle\nabla \Phi(u \vee v)-\nabla \Phi(v), u \vee v-v\rangle \\
& \quad \geq 0
\end{aligned}
$$

for any $u, v \in \mathfrak{X}$ (cf. [5; Proposition 1.2]). The assertions $\left(c_{1}^{\prime}\right) \rightarrow\left(c_{1}\right)$ and $\left(c_{3}\right) \rightarrow\left(a_{4}\right)$ are trivial, and $\left(c_{1}\right) \rightarrow\left(c_{2}\right)$ and $\left(c_{2}\right) \rightarrow\left(c_{3}\right)$ are known (cf. [13]).

\section{REFERENCES}

[1] H. Brezis and G. Stampacchia, Sur la régularité de la solution d'inéquations elliptiques, Bull. Soc. Math. France 96 (1968), 153-180.

[2] F. E. browder, On a theorem of Beurling and Livingston, Canad. J. Math. 17 (1965), 367-372.

[ 3 ] F. E. Browder, Nonlinear variational inequalities and maximal monotone mappings in Banach spaces, Math. Ann. 183 (1969), 213-231.

[4] B. Calvert, Nonlinear equations of evolution, Pacific J. Math. 39 (1971), 293-350.

[ 5 ] B. Calvert, Potential theoretic properties for nonlinear monotone operators, Boll. Un. Mat. Ital. 5 (1972), 473-489.

[6] M. G. Crandall and T. M. Liggett, Generation of semigroups of nonlinear transformations on general Banach spaces, Amer. J. Math. 93 (1971), 265-298. 
[ 7 ] J. Deny, Sur les espaces de Dirichlet, Sém. théorie du potentiel, Paris, 1957.

[ 8 ] M. Itô, A note on extended regular functional spaces, Proc. Japan Acad. 43 (1967), $435-440$.

[ 9 ] T. Kato, Accretive operators and nonlinear evolution equations in Banach spaces, Proc. Symp. Pure Math. A. M. S. 18, Part 1 (1970), 138-161.

[10] N. Kenmochi, Nonlinear operators of monotone type in reflexive Banach spaces and nonlinear perturbations, Hiroshima Math. J. 4 (1974), 229*263.

[11] N. Kenmochi and Y. Mizuta, The gradient of a convex function on a regular functional space and its potential theoretic properties, Hiroshima Math. J. 4 (1974), $743-763$.

[12] Y. Kōmura, Nonlinear semigroups in Hilbert space, J. Math. Soc. Japan 19 (1967), 493-507.

[13] Y. Konishi, Nonlinear semigroups in Banach lattices, Proc. Japan Acad. 47 (1971), 24-28.

N. KENMOCHI

Department of Mathematics

Faculty of Education

Chiba University

Chiba, Japan

Y. MIZUTA

Department of Mathematics

Faculty of Science

Hiroshima University

Hiroshima, Japan 